\title{
Comparative Transcriptome Analysis Depicts Candidate Genes Involved in Skin Color Differentiation in Red Tilapia
}

\author{
Jinzhao He ${ }^{1}$, Pengfei Feng ${ }^{2}$, Junqi Qin ${ }^{2}$, Yun Teng ${ }^{1}$, Xu Luo $^{2 *}$, Zigui Chen ${ }^{1 *}$, \\ Huawei $\mathrm{Ma}^{2 *}$ and Dayan Zhou ${ }^{1}$
}

${ }^{1}$ Breeding Laboratory, Guangxi Aquatic Products Introduction and Breeding Center, 30 Baisha Avenue, Jiangnan District, Nanning 530031, China.

${ }^{2}$ Biotechnology Laboratory, Guangxi Academy of Fishery Sciences, No. 8 Qingshan Road, Qingxiu District, Nanning 530021, China.

Jinzhao He, Pengfei Feng and Junqi Qin have contributed equally as first co-authors.

\section{A B S T R A C T}

This study aimed to investigate the genetic molecular mechanism of body color differentiation and variation of red tilapia, selecting the main genes related to the variation and cultivating the pure and stable red tilapia variety. The effects of different temperature treatments on body color and survival of Guam red tilapia, pearl white red tilapia and Florida red tilapia were compared. Besides, comparative transcriptome analysis was used to screen the candidate genes linked to the skin color differentiation of pearl white red tilapia. Among them, the body color of Guam red tilapia changed when the water temperature dropped to $16-14^{\circ} \mathrm{C}$, and continued to drop to about $11^{\circ} \mathrm{C}$, it was discolored in a large area reaching above $90 \%$. According to the differential analysis: Tyrosine Kinase STYK1, HSP70, HSP30 and Transcription factor Sp6 expressions were significantly increased in the low temperature group, while MC1R, Transcription factor (MafB, jun-D, AP-1, E2F5, ETV6, Sp9, Sp7, E2F1, Sp4) expressions were notably decreased. Further, the color variation of red tilapia at low temperature may result from the change of tyrosine activity in the body. The in-depth study of its regulatory mechanism contributes to understanding the genetic mechanism of red tilapia and improving the body shape.

\author{
Article Information \\ Received 26 March 2020 \\ Revised 14 December 2020 \\ Accepted 20 January 2021 \\ Available online 08 March 2022 \\ (early access)
}

Authors' Contribution

JH, PF and JQ presented the concept

of the study and wrote the manuscript. YT and XL designed the study, interpreted the results, wrote and revised the manuscript. $\mathrm{ZC}$ performed the experiments while DZ helped him. HM helped in writing and revising the manuscript.

Key words

Comparative transcriptome analysis,

Genetic molecular mechanism, Skin

color differentiation, Red tilapia,

Candidate genes for skin color

differentiation, Tyrosine Kinase,

HSP70, HSP30, Transcription factor

Sp6, Oreochromis spp.

\section{INTRODUCTION}

$\mathrm{R}$ ed Tilapia (genus Oreochromis), an essential tropical freshwater species is one of the most important edible fish in the world (Melo et al., 2014). It is widely cultured because of its rapid growth and tender meat (Noraini et $a l ., 2013)$.

Fish belongs to poikilothermic animals whose body temperature varies with the environmental temperature (Yang et al., 2004), water temperature affects the survival, growth, metabolism, reproduction of fish (Ross et al., 2013). Tilapia is a warm-water fish with a temperature tolerance of $6-42{ }^{\circ} \mathrm{C}$, but the tolerance to low temperature is influenced by water quality, fish age and health status (Nitzan et al., 2016). The extreme freezing weather in China always caused great economic loss to the tilapia farming industry (Luan, 2010). A larger number of red

\footnotetext{
* Corresponding author: 260229251@qq.com; 0czg@163.com; ma463543285@126.com 0030-9923/2022/0001-0001 \$ 9.00/0 Copyright 2022 Zoological Society of Pakistan
}

tilapia and wintering seedings that were ready for market were frozen and die under $8-10{ }^{\circ} \mathrm{C}$ of water temperature (Wang et al., 2018a). Additionally, the time of seedling release of the early spring red tilapia with low cold tolerance would be delayed, leading to its market price volatile (He et al., 2016). The supply and demand relationship of red tilapia was unbalanced, which had a major impact on the red tilapia breeding industry (El-Ebiary et al., 2013). Therefore, the physiological study on the cold tolerance of red tilapia can provide data supporting for the breeding of cold-resistant red tilapia in the future.

Differentiation during genetic selection and variation in body color has been a growing limitation to the commercial value of red tilapia (Wang et al., 2018b). The differentiation of body color in red tilapia is irreversible, while the change of skin color is reversible with the change of ambient temperature (Wang et al., 2018a). Fish pigment cells are mainly distributed in skin, and fins and scales are derivatives of the skin, and pigment cells are widely distributed. Previous study has shown that the two types of melanin produced by melanocytes extremely influence on body color phenotype, one of which is eumelanin 
being responsible for producing black and brown phenotypes, the other is brown melanin contributing to producing the yellow and red phenotypes (Kottler et al., 2015). The formation of melanin is a complex process requiring the maturation of melanocytes, the synthesis and transportation of melanin, in which regulatory factors and signaling molecules are involved in each stage (Poletini et al., 2016). The adenylate cyclase pathway, the protein kinase c pathway and the tyrosine pathway are three molecules and transduction pathways in melanocytes that synthesize melanin (Higanakamine et al., 2015). The common genetic signaling pathways of two pigments have been discovered (Mandal et al., 2010). The eumelanin is synthesized from tyrosine in vivo. Under the action of tyrosinase, tyrosine forms a eumelanin through a series of oxidation and catalytic polymerization (Chodurek et al., 2013). It turns to the synthetic pathway of brown melanin after the reduction of tyrosinase activity (Chodurek et al., 2013). Compared with other fish, little is known to the body color differentiation in red tilapia. Zhu et al. (2016) used comparative transcriptome sequencing to find the following genes related to skin color in red tilapia of three different colors: tyr, tyrp1, silv, sox 10, slc24a5, CBS and slc7a11 (Zhu et al., 2016). The results showed that the miRNAs associated with red tilapia color mainly included slc7a11, mclr and asip, predicating that miR-138-5p and miR-722 played an important role in the regulation of pigmentation. However, the molecular mechanism of body color differentiation remains unclear.

Thus, in this study, transcriptome sequencing was performed on the tissue samples of pearly white tilapia fin strips at low temperature and normal temperature to screen out the genes related to body color variation of red tilapia and provide a theoretical basis for the breeding of fine red tilapia strains.

\section{MATERIALS AND METHODS}

\section{Sample collection}

Thirtysamples, each of Guam red tilapia (GR), pearl white red tilapia (WR) and Florida red tilapia (FR), which were bred in the same year and weighed $10 \mathrm{~g}, 80 \mathrm{~g}$ and $120 \mathrm{~g}$, respectively were selected for the experiment. The animal experiment complying with the ARRIVE guidelines was carried out in accordance with the U.K. Animals (Scientific Procedures) Act, 1986 and associated guidelines, EU Directive 2010/63/EU for animal experiments. Different red tilapia strains were placed in diverse aquarium for 5 days at $20^{\circ} \mathrm{C}$ to adapt to the living environment of the aquarium for subsequent temperature culture test. Fish were fasted for $24 \mathrm{~h}$ before the trial. The circulating water filtration system was adopted in this experiment, and feeding was conducted at 9:00 am and 4:00 pm, respectively every day. The laboratory temperature was artificially cooled, from $20{ }^{\circ} \mathrm{C}$ to $6{ }^{\circ} \mathrm{C}$ with gradient of $0.2^{\circ} \mathrm{C} / 2 \mathrm{~h}$. During this period, water temperature was recorded every $2 \mathrm{~h}$ during the day and every $4 \mathrm{~h}$ at night. Meanwhile, data such as the deaths, the temperature at which the body color began to change, the location and degree of discoloration of red tilapia in each group were recorded. In the normal water temperature $\left(20^{\circ} \mathrm{C}\right)$ and cooling process (after $24 \mathrm{~h}$ of body color change), 3 fin tissues of WR were extracted for transcriptome sequencing respectively, stored in a $-80{ }^{\circ} \mathrm{C}$ freezer before sequencing.

\section{RNA isolation and cDNA library construction}

Total RNA was extracted from tissue samples using MagZol Reagent. Then concentration and mass of each RNA sample was detected by nucleic acid protein detector (OD260: OD280) (Eppendorf, Germany) and agarose gel electrophoresis, respectively. The qualified RNA samples were enriched with magnetic beads with Oligo (dT). cDNA strand was synthesized with six base primers using enriched mRNA as template, and cDNA library was obtained by a series of modifications and PCR enrichment. The cDNA library was then quantitatively analyzed by Qbuit3.0, and the library insert size was detected by Agilent 2100 Bioanalyzer to ensure that the library effective concentration and quality meet the requirements of the RNA sequencing.

Transcriptome sequencing and de novo assembly of transcriptome

Each qualified cDNA library was sequenced using Illumina HiSeq2500 for $2 \times 125$ bp pair-end (PE) sequencing. Quality control of all raw reads was conducted by Fastqc (http://www.bioinformatics.babraham.ac.uk/ projects/fastqc/) software. Raw data obtained from sequencing were filtered, and connector sequences and low-quality reads were removed to obtain high quality clean data. Gene assembly analysis was performed on high quality clean data after quality control. Trinity (v2.2.0) analysis software based on DE Bruijn graphs was used for subsequent analysis to assemble the transcriptome sequencing data from scratch. The sequences output from Trinity software can be used for further analysis.

\section{Assembled sequence annotation: Functional annotation}

Functional annotation of the assembled sequences was performed by homology searches against the Uni ProtSwiss Prot (The Universal Protein Resource) database, the GO (Gene Ontology) database, the KEGG (Kyoto Encyclopedia of Genes and Genomes) database and the TrEMBL protein database, and searches were conducted 
by the BLAST program.

\section{RT-PCR analysis}

Total RNA was isolated from samples by TRIzol method (Invitrogen, CA, USA), and cDNA was obtained by reverse transcription after DNA elimination. The results were calculated by ABI Prism 7500 RT-PCR system (Applied Biosystem, Foster City, CA, USA). Amplification was performed in a mixture $(10 \mu \mathrm{l})$ containing $5 \mu \mathrm{l}$ of $2 \mathrm{X}$ SYBR Green Master Mix, $1 \mu \mathrm{l}$ of cDNA and $0.2 \mu \mathrm{l}$ of each primer $(10 \mu \mathrm{M})$. The thermal cycling curve consisted of an initial denaturation at $95^{\circ} \mathrm{C}$ for $5 \mathrm{~min}$, followed by 40 cycles of $95{ }^{\circ} \mathrm{C}$ for $15 \mathrm{~s}$, annealing/extension at $60{ }^{\circ} \mathrm{C}$ for $45 \mathrm{~s}$. Melting curve was generated by heating to $95^{\circ} \mathrm{C}$ and cooling to $65^{\circ} \mathrm{C}$. All reactions were performed in triplicate and included a negative control without template. The value is based on identifying two biological replicates, each repeated 3 times. The gene expression level was normalized to $\beta$-actin expression in the same sample. A two-tailed t-test was used to compare expression levels.

\section{RESULTS}

Effect of temperature on apparent body color of red tilapia

When the temperature dropped to $15^{\circ} \mathrm{C}$, body color of the PR and FR with the various body weight began to change; body color of the PR and FR changed over large areas when the temperature was decreased to $11^{\circ} \mathrm{C}$, and the discoloration ratio is all over $90 \%$. When the temperature dropped to $10.2{ }^{\circ} \mathrm{C}$, GR (10 g) began to change color, as temperature continued to reduce, the total body color change ratio was about $6 \%$. However, for GR (80 g and $120 \mathrm{~g})$, the decrease of temperature has no effect on the change of body color (Table I).

Table I. Changes of body color of different species of red tilapia at different temperatures.

\begin{tabular}{lllll}
\hline Weight & Species & $\mathbf{T}_{\mathbf{1}}\left({ }^{\circ} \mathbf{C}\right)$ & $\mathbf{T}_{\mathbf{2}}\left({ }^{\circ} \mathbf{C}\right)$ & Discoloration ratio (\%) \\
\hline $10 \mathrm{~g}$ & $\mathrm{~A}$ & 10.2 & 0 & 6 \\
& $\mathrm{~B}$ & $15 \sim 13$ & $11 \sim 9$ & 91 \\
& $\mathrm{C}$ & $14 \sim 13$ & $11.5 \sim 9.6$ & 95 \\
$80 \mathrm{~g}$ & $\mathrm{~A}$ & 0 & 0 & 0 \\
& $\mathrm{~B}$ & $15 \sim 14$ & $11.6 \sim 9.8$ & 94 \\
& $\mathrm{C}$ & $16 \sim 15$ & $12.1 \sim 9.8$ & 97 \\
$120 \mathrm{~g}$ & $\mathrm{~A}$ & 0 & 0 & 0 \\
& $\mathrm{~B}$ & $16 \sim 14$ & $11.8 \sim 10.3$ & 96 \\
& $\mathrm{C}$ & $16 \sim 15$ & $11.8 \sim 10.0$ & 97 \\
\hline
\end{tabular}

$\mathrm{T} 1$, The water temperature starting to change color; $\mathrm{T} 2$, Water temperature when large areas of color changing. (A) Guam red tilapia; (B) Pearl white red tilapia; (C) Florida red tilapia.
Transcriptome sequencing and assembly

In the gradient cooling culture process, the apparent body color of WR changed obviously. Thus, 3 fin tissues of WR in the normal water temperature $\left(20{ }^{\circ} \mathrm{C}\right)$ and cooling process (after $24 \mathrm{~h}$ of body color change) were selected for transcriptome sequencing. In total, a mean of 8, 194, 153 paired-end (PE) clean reads for each library were obtained after data filtering and the sequencing quality was high with Q30 ratio larger than 96\% for all samples (Table II).

We pooled all the clean reads from six libraries and de novo assembled them using Trinity. A total of 117,609 contigs, corresponding to unique genes of which length ranged from $201 \mathrm{bp}$ to $9,868 \mathrm{bp}$. There were 27,273 $(23.19 \%)$ contigs longer than $1,000 \mathrm{bp}$. The detailed length distribution of transcripts from the red tilapia is shown in Figure 1.

Table II. Transcriptome sequencing data of red tilapia.

\begin{tabular}{|c|c|c|c|c|c|}
\hline $\begin{array}{l}\text { Sam- } \\
\text { ple }\end{array}$ & $\begin{array}{l}\text { Number of } \\
\text { clean reads }\end{array}$ & $\begin{array}{l}\text { Number of } \\
\text { bases }\end{array}$ & $\begin{array}{l}\text { Length } \\
\text { (bp) }\end{array}$ & $\begin{array}{l}\text { GC } \\
(\%)\end{array}$ & Q20 Q30 \\
\hline HLF3 & 8213990 & 1026748750 & 125 & 48.57 & 98.9398 .02 \\
\hline HLF7 & 8174316 & 1021789500 & 125 & 48.58 & 97.8996 .2 \\
\hline $\begin{array}{l}\text { Read_nu } \\
\text { bases se } \\
\text { base and } \\
\text { having a }\end{array}$ & d & $\begin{array}{l}\text { quenc } \\
\text { (bp), }\end{array}$ & 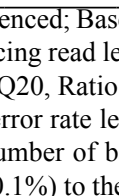 & $8 \mathrm{nu}$ & $\begin{array}{l}\text { ber of } \\
\text { o of G } \\
\text { bases } \\
\text { e total }\end{array}$ \\
\hline
\end{tabular}

\section{$G O$ annotation and analysis of DEGS}

Gene ontology (GO) annotation was then performed based on the $\mathrm{Nr}$ annotation and 34,542 contigs (29.37\%) were assigned to GO term (Fig. 2). As shown in Figure 2, a total of 49 terms were assigned, including 23 (46.94\%) biological process category, 13 (26.53\%) cellular component terms and $13(26.53 \%)$ molecular function terms. In the biological process category, cellular process was the most abundant term, followed by metabolic process biological regulation and pigmentation. For the cellular component category, cell and cell part were the predominant terms which were followed by organelle. For the molecular function category, binding was the main term, and it was followed by catalytic molecular transducer and transcription regulator (Fig. 2).

\section{Functional annotation}

All contigs were compared with four data bases including EMBL database, the UniProt-SwissProt database, KEGG database and GO database for functional annotation. There were 27997, 35370, 34542 assembled contigs that had significant hits against EMBL database, the UniProt-SwissProt database and GO database, 
length distribution of transcripts from the red tilapia

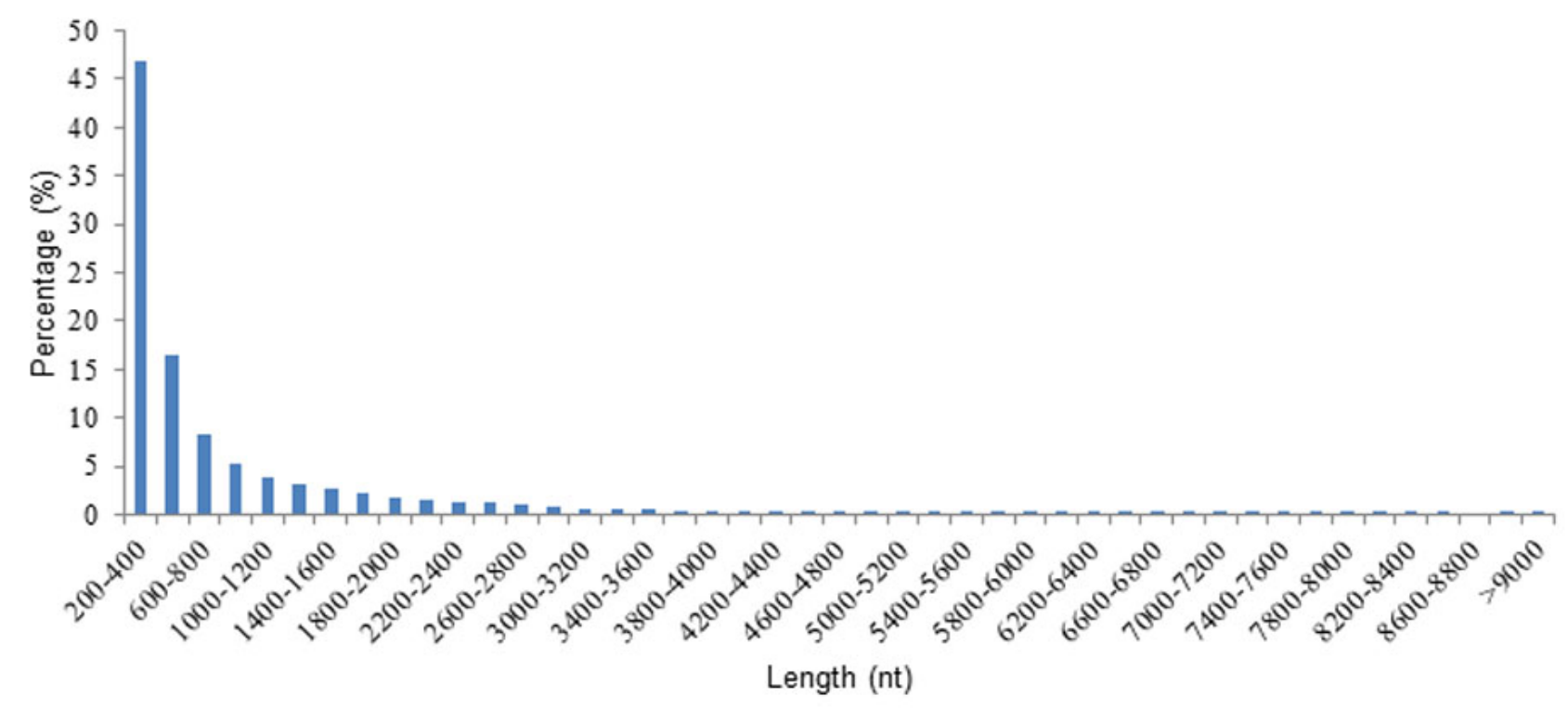

Fig. 1. Transcriptome sequencing and assembly. The detailed length distribution of transcripts from the red tilapia.

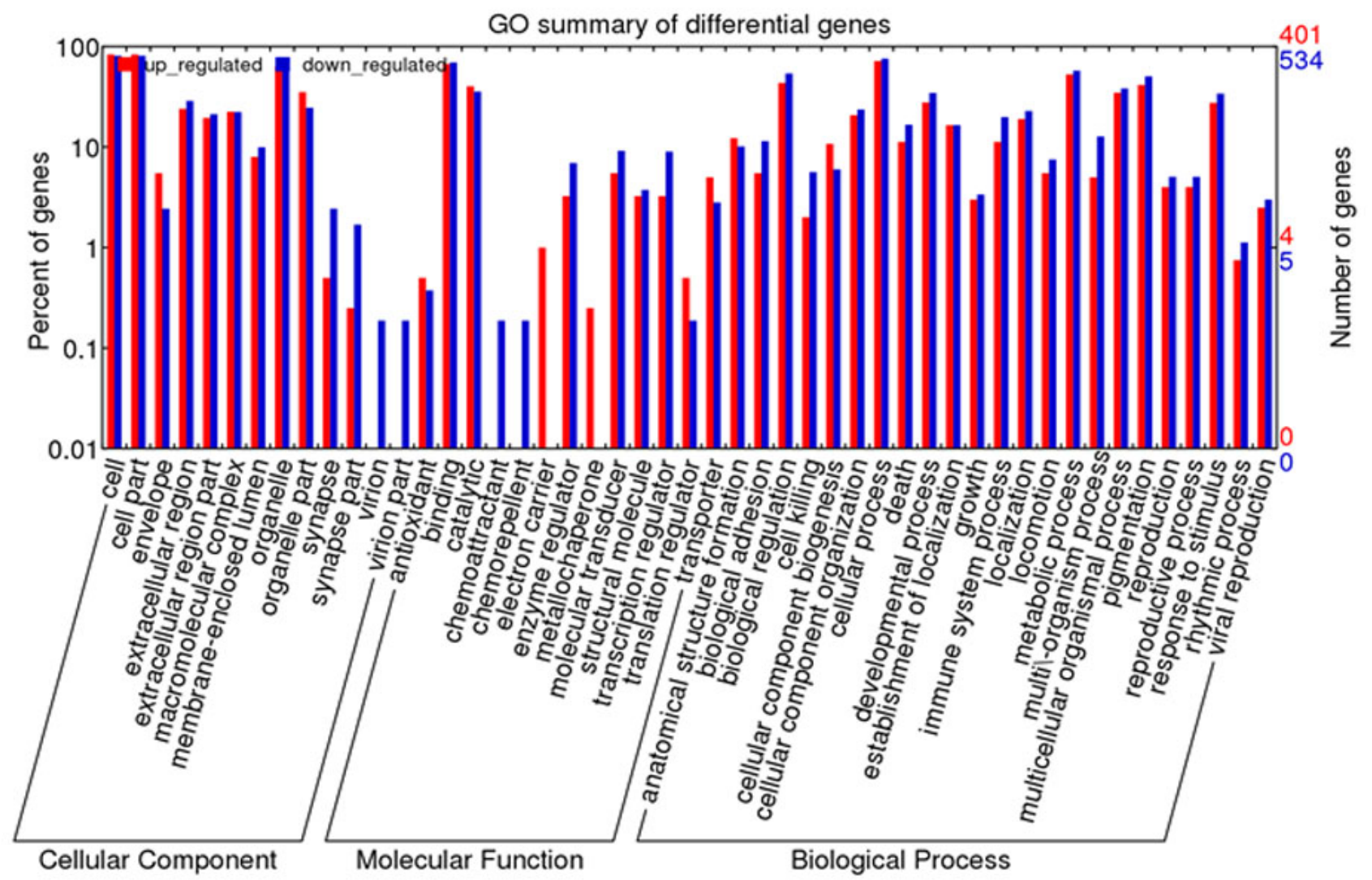

Fig. 2. GO annotation. Gene ontology classification of contigs. 
respectively (Table III, Fig. 2). To identify the biological pathway in the red tilapia transcriptome, all contigs were mapped to the KEGG database and were associated with 216 pathways (Supplementary Table I).

\section{Table III. Statistics of function annotation.}

\begin{tabular}{ll}
\hline Annotated database & Annotated number \\
\hline Swiss-PROT & 35370 \\
EMBL & 27997 \\
GO & 34542 \\
All contigs & 117609 \\
\hline
\end{tabular}

Identifying candidate genes involved in skin color and pigmentation

To show the differences in the skin color of red tilapia, we made a comparative analysis of two skin transcriptomes. Based on the standard that $|\log \mathrm{FC}| \geq 1$ and FDR $\leq 0.5,3,228$ differentially expressed transcripts were identified between HLF7 and HLF3, which include 1,327 up-regulated transcripts and 1,901 down-regulated transcripts in HLF7. Candidate gene enrichment analysis identified 49 candidate genes involved in the processes of skin color and pigmentation from the two transcriptome data sets. Interestingly, six genes were revealed to be specifically expressed in HLF7, while 16 genes were only found expressed in HLF3 (Table IV).

\section{DISCUSSION}

We employed the transcriptome analysis to investigate the different gene expression of fin tissues of WR, indicating that the color change of red tilapia at low temperature arose from the co-regulation of multiple genes (tyrosine protein kinase STYK1, HSP 70, HSP 30, HSP 90, $M C 1 R$ and transcription factor).

Heat shock protein (HSP70) is one of the most conservative and important proteins in HSPP family which is synthesized by the reaction of organism to the physical, chemical and biological stress agents in the environment (Shen et al., 2016). HSP70 gene can produce abundant heat stress proteins, remove the denatured proteins, protect the cells from the damage of denatured proteins, and reduce the impact of external stress environment on the body of fish when the cells are under stress (González-Aravena et al., 2018). Studies have found that the HSP70 mRNA expression in the liver was significantly increased after 12 $\mathrm{h}$ of low-temperature stress on the fish (Ming et al., 2012). It has been demonstrated that both low temperature and high temperature caused the rapid expression of HSP70 gene in the body cells of platy bream and the synthesis of heat stress protein (Ming et al., 2012). In our study, the expression of HSP70 in HLF7 cultured at low temperature was significantly higher than that in normal temperature group, suggesting that the low temperature environment outside led to the changes in the physiological function of red tilapia. Besides, it is worth noting that the fry that has not yet fully developed are more susceptible to temperature changes and change their body color in our experiments, such as GR. Consistently, studies have shown that huge genetic variation in body color can provide a good indication of its potential improvement, and the body length and width of red tilapia are negatively correlated with body color (-0.47 to -0.25) (Hamzah et al., 2017). However, the specific mechanism of this change still needs further study.

The biological basis of body color change in fish is the number, morphology and distribution of different pigment cells on skin and scales (Steffen et al., 2015). Compared with only a kind of pigment cell in the mammalian (melanocytes), bony fishes were found to have 6 kinds of pigment cells, including the melanocytes, red pigment cells, yellow pigment cells, rainbow, white and blue pigment cells, making the formation and changes of the fish body color more complicated and different red tilapia showing different body colors (Erickson, 2010). It has also been reported that MC1R gene, ASIP gene and $T Y R P 1$ gene are the major candidate genes of fish body color formation (Jian-Xiong et al., 2014). MC1R gene, as a key gene to produce melanin, expressed slightly in HLF7 of the low temperature group, and it was not the main reason for the formation of black spots (San-Jose et $a l ., 2015)$. It is speculated that the formation of melanin by $M C 1 R$ gene was affected by the albino gene. Meanwhile, the $M C 1 R$ gene, which was related to the synthesis of melanin, represents a critical role in the formation of fish body color. However, MC1R expression was low in HLF7 in black-spotted red tilapia of the low-temperature group in our study, probably because it was an acute regulator and made pigments significantly deposited in the short term. And hence the surface of the skin was colored with streaks, and then black markings or red markings, but the expression level was stable.

It has been revealed that the body color of Mozambique tilapia became black in the dark after $25 \mathrm{~h}$, but there was no significant change in MC1R expression in the epidermis and the $\alpha-\mathrm{MSH}$ level in the serum. Therefore, the $M C 1 R$ gene could also act as an acute regulator in the regulation of fish pigmentation, but the pigment is significantly deposited in a short period sine this activity expression cannot be maintained for a long time (Moorman et al., 2015). So even if the red tilapia epidermis exhibited a certain color, there was no significant change in MC1R mRNA expression in fish tissues. 
Table IV. Expression and annotation of candidate genes involved in skin color and pigmentation between HLF7 and HLF3.

\begin{tabular}{|c|c|c|c|c|}
\hline Gene_ID & FPKMHLF3 & $\begin{array}{l}\text { FPKM } \\
\text { HLF7 }\end{array}$ & $\begin{array}{l}\text { Fold change } \\
(\log 2)\end{array}$ & Annotation \\
\hline TRINITY_DN24220_c0_g4 & 0 & 62.65 & 10.41823029 & Heat shock protein 30 \\
\hline TRINITY_DN24220_c0_g1 & 0 & 62.17 & 10.0705945 & Heat shock protein 30 \\
\hline TRINITY_DN45765_c0_g1 & 0 & 19.36 & 9.377526023 & Heat shock protein HSP 90-alpha \\
\hline TRINITY_DN24220_c0_g2 & 0 & 61.72 & 8.461914888 & Heat shock protein 30 \\
\hline TRINITY_DN24220_c0_g3 & 0 & 55.3 & 8.292501236 & Heat shock protein 30 \\
\hline TRINITY_DN22422_c0_g1 & 0.49 & 50.84 & 8.0814764 & Heat shock $70 \mathrm{kDa}$ protein 1 \\
\hline TRINITY_DN2485_c0_g1 & 0 & 1.36 & 7.204597264 & Transcription factor Sp6 \\
\hline TRINITY_DN40960_c0_g1 & 2.04 & 61.57 & 5.358205112 & Heat shock protein HSP 90-beta \\
\hline TRINITY_DN55897_c0_g1 & 1.4 & 21.72 & 4.455123638 & Heat shock protein HSP 90 -alpha \\
\hline TRINITY_DN290_c0_g1 & 0.33 & 3.55 & 3.922387454 & Heat shock protein beta- 8 \\
\hline TRINITY_DN7424_c0_g1 & 0.45 & 4.57 & 3.839197418 & Myosin-binding protein $\mathrm{C}$, fast-type \\
\hline TRINITY_DN30976_c0_g1 & 4.41 & 38.63 & 3.665320161 & Heat shock protein HSP 90-alpha \\
\hline TRINITY_DN45441_c0_g3 & 0.23 & 2.1 & 3.523276762 & Mitogen-activated protein kinase 8 \\
\hline TRINITY_DN21271_c0_g1 & 2.58 & 12.4 & 2.880544153 & Tyrosine-protein kinase STYK1 \\
\hline TRINITY_DN44078_c0_g1 & 2.11 & 10.63 & 2.861156424 & Heat shock protein HSP 90-alpha \\
\hline TRINITY_DN20921_c0_g1 & 13.79 & 67.1 & 2.829661276 & Carbonic anhydrase 4 \\
\hline TRINITY_DN12210_c0_g1 & 60.47 & 219.48 & 2.40838373 & Dual specificity protein phosphatase 5 \\
\hline TRINITY_DN10113_c0_g1 & 1.69 & 7.21 & 2.37892893 & Cyclin-dependent kinase 16 \\
\hline TRINITY_DN23272_c0_g2 & 5.5 & 18.25 & 2.278654253 & Heat shock $70 \mathrm{kDa}$ protein 4 \\
\hline TRINITY_DN11134_c0_g1 & 219.16 & 30.3 & -2.305170585 & Transcription factor jun-D \\
\hline TRINITY_DN19725_c1_g2 & 14.92 & 1.89 & -2.426863715 & Fibroblast growth factor receptor 1-A \\
\hline TRINITY_DN19383_c0_g1 & 0.48 & 0 & -2.558910746 & Sphingosine 1-phosphate receptor 1 \\
\hline TRINITY_DN25429_c2_g1 & 7.02 & 0.74 & -2.644540997 & $\begin{array}{l}\text { Calcium/calmodulin-dependent protein kinase type II } \\
\text { subunit gamma }\end{array}$ \\
\hline TRINITY_DN5260_c0_g1 & 21.2 & 2.24 & -2.686316481 & Protein kinase $\mathrm{C}$ beta type \\
\hline TRINITY_DN22985_c0_g1 & 32.56 & 4.14 & -2.716853168 & Dual specificity protein phosphatase 2 \\
\hline TRINITY_DN24675_c0_g2 & 11.08 & 1.16 & -2.731113656 & Myosin light chain kinase, smooth muscle \\
\hline TRINITY_DN25683_c1_g3 & 5 & 0.37 & -2.789473925 & Heat shock $70 \mathrm{kDa}$ protein $12 \mathrm{~A}$ \\
\hline TRINITY_DN15797_c0_g1 & 1.23 & 0 & -2.805596395 & Serine/threonine-protein kinase N1 \\
\hline TRINITY_DN20404_c0_g1 & 4.67 & 0.36 & -3.138131195 & Mast/stem cell growth factor receptor Kit \\
\hline TRINITY_DN5929_c0_g1 & 295.71 & 22.79 & -3.148401717 & Transcription factor jun-D \\
\hline TRINITY_DN1493_c0_g1 & 4.86 & 0.36 & -3.168662628 & Epithelial discoidin domain-containing receptor 1 \\
\hline TRINITY_DN2926_c0_g1 & 5.24 & 0.36 & -3.272375487 & Traf2 and NCK-interacting protein kinase \\
\hline TRINITY_DN11441_c0_g1 & 196.91 & 9.25 & -3.862108956 & Transcription factor AP-1 \\
\hline TRINITY_DN20977_c0_g1 & 5.43 & 0 & -4.447806609 & Transcription factor E2F5 \\
\hline TRINITY_DN63906_c0_g1 & 4.11 & 0.11 & -4.585107644 & Transcription factor $\mathrm{Sp} 7$ \\
\hline TRINITY_DN9373_c0_g1 & 6.33 & 0 & -6.800394984 & $\begin{array}{l}\text { Hormonally up-regulated neu tumor-associated kinase } \\
\text { homolog A }\end{array}$ \\
\hline TRINITY_DN25415_c0_g1 & 1.25 & 0 & -7.019203017 & Collagen alpha-1(XII) chain \\
\hline
\end{tabular}




\begin{tabular}{lllll}
\hline Gene_ID & FPKMHLF3 & $\begin{array}{l}\text { FPKM } \\
\text { HLF7 }\end{array}$ & $\begin{array}{l}\text { Fold change } \\
\text { (log2) }\end{array}$ & Annotation \\
\hline TRINITY_DN71751_c0_g1 & 2.12 & 0 & -7.09264739 & Transcription factor E2F1 \\
TRINITY_DN9015_c0_g1 & 6.62 & 0 & -7.09264739 & Transcription factor Sp4 \\
TRINITY_DN3355_c0_g1 & 1.84 & 0 & -7.09264739 & STE20-related kinase adapter protein beta \\
TRINITY_DN53770_c0_g1 & 3.26 & 0 & -7.09264739 & Ephrin type-A receptor 7 \\
TRINITY_DN8410_c1_g1 & 3.07 & 0 & -7.353919053 & Heat shock 70 kDa protein 12A \\
TRINITY_DN4370_c0_g1 & 10.21 & 0 & -7.468717718 & Heat shock 70 kDa protein 12B \\
TRINITY_DN25415_c0_g2 & 1.93 & 0 & -7.625416211 & Collagen alpha-1(XII) chain \\
TRINITY_DN25683_c0_g1 & 12.05 & 0 & -8.122847214 & Heat shock 70 kDa protein 12A \\
TRINITY_DN5021_c0_g1 & 4.83 & 0 & -8.670545959 & Transcription factor Sp9 \\
TRINITY_DN43769_c0_g1 & 100.52 & 0.14 & -8.913081177 & Neoverrucotoxin subunit alpha \\
TRINITY_DN24960_c0_g3 & 26.53 & 0 & -9.961230712 & Transcription factor ETV6 \\
TRINITY_DN1001_c0_g1 & 22.66 & 0 & -11.23238536 & Transcription factor MafB \\
\hline
\end{tabular}

Tyrosinase is the rate-limiting enzyme of melanin synthesis vital for the rate and quantity of eumelanin and pheomelanin (Hirobe et al., 2011). During the formation of melanin, hormones, inorganic ions which are in vivo and external light, UV light physicochemical factors may affect the activity of TYR. Besides, the of the eumelanin to pheomelanin by $A S I P$ gene was linked to the expression of the tyrosine family gene (TYR, TYRP1, DCT) and reduced its expression (Meng et al., 2014). Currently, the expression of gene related to the tyrosinase synthesis becomes an important indirect indicator of the energy of melanin formation in fish due to the absence of an effective method for determining the melanin content and body color index of fish (Chen et al., 2012). In addition, the coding transcription factors of pigment cells are also regulated by gene signal channels. It has been found that gene knockout can regulate the transcription factors of pigment cells in c-kit signal transduction, resulting in the loss of their function and pigmentation (Otsuki et al., 2020). Low temperature stress can also lead to changes in the expression of transcription factors. In our study, the expression of transcription factors decreased significantly at low temperature, but the related mechanism is unknown.

\section{CONCLUSION}

In this study, the body color of the GR with a small body weight changed significantly with temperature, and the melanin synthesis ability was enhanced in the low temperature group HLF7. The result was consistent with the tendency of red tilapia to become darker as the temperature gradient decreased. The pathway of melanin synthesis is a complex process involving the participation and regulation of polygene. Nevertheless, the overall melanin content, the ratio of eumelanin to brown melanin and the molecular metabolic mechanism of red tilapia body color variation was still needed to be further studied.

\section{ACKNOWLEDGEMENTS}

This study was financially supported by National Modern Agricultural Industry Technology System Guangxi Tilapia Innovation Team (No. 2016LFY05), Guangxi Science and Technology Base and Specialized Talents (AD17195016, AD18294012).

\section{Supplementary material}

There is supplementary material associated with this article. Access the material online at: https://dx.doi. org/10.17582/journal.pjz/20200326090308

\section{Statement of conflict of interest}

The authors have declared no conflict of interest.

\section{REFERENCES}

Chen, W., Wang, H., Dong, B., Dong, Z., Zhou, F., Fu, Y. and Zeng, Y., 2012. Molecular cloning and expression analysis of tyrosinase gene in the skin of Jining Gray Goat (Capra hircus). Mol. Cell Biochem., 366: 11-20. https://doi.org/10.1007/ s11010-012-1275-1

Chodurek, E., Zdybel, M. and Pilawa, B., 2013. Application of EPR spectroscopy to examination of free radicals in melanins from A-375 and G-361 human melanoma malignum cells. J. appl. Biomed., 11: 173-185. https://doi.org/10.2478/v10136-012- 
0023-X

El-Ebiary, E.H., Wahbi, O.M. and El-Greisy, Z.A., 2013. Influence of dietary Cadmium on sexual maturity and reproduction of red tilapia. Egypt $J$. aquat. Res., 39: 313-317. https://doi.org/10.1016/j. ejar.2013.12.005

Erickson, C.A., 2010. From the crest to the periphery: Control of pigment cell migration and lineage segregation. Pigment Cell Res., 6: 336-347. https:// doi.org/10.1111/j.1600-0749.1993.tb00611.x

González-Aravena, M., Calfio, C., Mercado, L., Morales-Lange, B., Bethke, J., Lorgeril, J.D. and Cárdenas, C.A., 2018. HSP70 from the Antarctic sea urchin Sterechinus neumayeri: Molecular characterization and expression in response to heat stress. Biol. Res., 51: 8. https://doi.org/10.1186/ s40659-018-0156-9

Hamzah, A., Thoa, N.P. and Nguyen, N.H., 2017. Genetic analysis of a red tilapia (Oreochromis spp.) population undergoing three generations of selection for increased body weight at harvest. $J$. appl. Genet., 58: 509-519. https://doi.org/10.1007/ s13353-017-0411-8

He, Y.F., Wang, L.M., Zhu, W.B., Dong, Z.J. and Liu, N., 2016. Effects of salinity on cold tolerance of Malaysian red tilapia. Aquacult. Int., 25: 1-16. https://doi.org/10.1007/s10499-016-0077-y

Higanakamine, S., Maeda, N., Toku, S. and Yamamoto, H., 2015. Involvement of protein kinase D1 in signal transduction from the protein kinase $\mathrm{C}$ pathway to the tyrosine kinase pathway in response to gonadotropin-releasing hormone. J. biol. Chem., 290: 25974-25985. https://doi.org/10.1074/jbc. M115.681700

Hirobe, T., Ito, S. and Wakamatsu, K., 2011. The mouse pink-eyed dilution allele of the P-gene greatly inhibits eumelanin but not pheomelanin synthesis. Pigment Cell Melanoma Res., 24: 241-246. https:// doi.org/10.1111/j.1755-148X.2010.00783.x

Jian-Xiong, Y.U., Zhang, Y.L., Zhou, Z.J., Wang, F.J., Wang, A.G., Wang, J.Y., Center, L.A. and University, D.M., 2014. Investigation of coat color gene MC1R (R306ter) and TYRP1 (Q331ter) SNP loci in labrador and golden retriever. Lab. Anim. Sci., 31: 37-41.

Kottler, V.A., Künstner, A. and Schartl, M., 2015. Pheomelanin in fish? Pigment Cell Melanoma Res., 28: 355-356. https://doi.org/10.1111/pcmr.12359

Luan, D.T., 2010. Genetic studies of Nile tilapia (Oreochromis niloticus) for farming in northern Vietnam: growth, survival and cold tolerance in different farm environments. $\mathrm{PhD}$ thesis, Norwegian
University of Life Sciences, Ås, Norway.

Mandal, D., Fu, P. and Levine, A.D., 2010. REDOX regulation of IL-13 signaling in intestinal epithelial cells: usage of alternate pathways mediates distinct gene expression patterns. Cell Signal, 22: 14851494. https://doi.org/10.1016/j.cellsig.2010.05.017

Melo, K.M., Grisolia, C.K., Pieczarka, J.C., de Souza, L.R., Filho, J.S. and Nagamachi, C.Y., 2014. FISH in micronucleus test demonstrates aneugenic action of rotenone in a common freshwater fish species, Nile tilapia (Oreochromis niloticus). Mutagenesis, 29: 215. https://doi.org/10.1093/mutage/geu005

Meng, H., Xu, R., Rong, D., Hui, L., Li, L., Wan, P. and Shi, G., 2014. Research progress of genes related to melanin synthesis in sheep. Biotechnol. Bull., 46: 34-39.

Ming, J., Xie, J., Xu, P., Ge, X., Liu, W. and Ye, J., 2012. Effects of emodin and vitamin $C$ on growth performance, biochemical parameters and two HSP70s mRNA expression of Wuchang bream (Megalobrama amblycephala Yih) under high temperature stress. Fish Shellf. Immunol., 32: 651661. https://doi.org/10.1016/j.fsi.2012.01.008

Moorman, B.P., Lerner, D.T., Grau, E.G. and Seale, A.P., 2015. The effects of acute salinity challenges on osmoregulation in Mozambique tilapia reared in a tidally changing salinity. $J$. exp. Biol., 218: 731739. https://doi.org/10.1242/jeb.112664

Nitzan, T., Slosman, T., Gutkovich, D., Weller, J.I., Hulata, G., Zak, T., Benet, A. and Cnaani, A., 2016. Maternal effects in the inheritance of cold tolerance in blue tilapia (Oreochromis aureus). Environ. Biol. Fish, 99: 975-981. https://doi.org/10.1007/s10641016-0539-0

Noraini, O., Sabri, M.Y. and Sitizahrah, A., 2013. Efficacy of spray administration of formalin-killed Streptococcus agalactiae in hybrid Red Tilapia. $J$. aquat. Anim. Hlth., 25: 142-148. https://doi.org/10. 1080/08997659.2013.781553

Otsuki, Y., Okuda, Y., Naruse, K. and Saya, H., 2020. Identification of kit-ligand a as the gene responsible for the medaka pigment cell mutant few melanophore. G3 (Bethesda), 10: 311-319. https://doi.org/10.1534/g3.119.400561

Poletini, M.O., de Assis, L.V., Moraes, M.N. and Castrucci, A.M., 2016. Estradiol differently affects melanin synthesis of malignant and normal melanocytes: A relationship with clock and clockcontrolled genes. Mol. Cell Biochem., 421: 1-11. https://doi.org/10.1007/s11010-016-2781-3

Ross, S.D., Behrens, J.W., Brander, K., Methling, C. and Mork, J., 2013. Haemoglobin genotypes in cod 
(Gadus morhua L): Their geographic distribution and physiological significance. Comp. Biochem. Physiol. A Mol. Integr. Physiol., 166: 158-168. https://doi.org/10.1016/j.cbpa.2013.05.025

San-Jose, L.M., Ducrest, A.L., Ducret, V., Béziers, P., Simon, C., Wakamatsu, K. and Roulin, A., 2015. Effect of the MC1R gene on sexual dimorphism in melanin-based colorations. Mol. Ecol., 24: 27942808. https://doi.org/10.1111/mec.13193

Shen, Q., Zhao, L., Xie, G., Ping, W., Yang, M., Wang, S., Fan, Z. and Tang, B., 2016. Cloning three Harmonia axyridis (Coleoptera: Coccinellidae) heat shock protein 70 family genes: Regulatory function related to heat and starvation stress. J. entomol. Sci., 50: 168-185. https://doi.org/10.18474/JES14-30.1

Steffen, J.E., Learn, K.M., Drumheller, J.S., Boback, S.M. and Mcgraw, K.J., 2015. Carotenoid composition of colorful body stripes and patches in the painted turtle (Chrysemys picta) and red-eared slider (Trachemys scripta). Chelonian Conserv. Biol., 14: 56-63. https://doi.org/10.2744/ccab-1401-56-63.1
Wang, L., Song, F., Zhu, W., Dong, J., Jianjun, F.U. and Dong, Z., 2018a. Effects of temperature on body color of Malaysian red tilapia during overwintering period. J. Fish. China, 42: 72-79.

Wang, L., Zhu, W., Dong, Z., Song, F., Dong, J. and Fu, J., 2018b. Comparative microRNA-seq analysis depicts candidate miRNAs involved in skin color differentiation in Red Tilapia. Int. J. mol. Sci., 19: 1209. https://doi.org/10.3390/ijms19041209

Yang, Y., Xie, S., Lei, W., Zhu, X. and Yang, Y., 2004. Effect of replacement of fish meal by meat and bone meal and poultry by-product meal in diets on the growth and immune response of Macrobrachium nipponense. Fish Shellf. Immunol., 17: 105-114. https://doi.org/10.1016/j.fsi.2003.11.006

Zhu, W., Wang, L., Dong, Z., Chen, X., Song, F., Liu, N., Yang, H. and Fu, J., 2016. Erratum: Comparative transcriptome analysis identifies candidate genes related to skin color differentiation in Red Tilapia. Sci. Rep., 6: 31347. https://doi.org/10.1038/ srep 31347 


\title{
Supplementary Material
}

\section{Comparative Transcriptome Analysis Depicts Candidate Genes Involved in Skin Color Differentiation in Red Tilapia}

\author{
Jinzhao He${ }^{1}$, Pengfei Feng², Junqi Qin², Yun Teng ${ }^{1}$, Xu Luo ${ }^{2 *}$, Zigui Chen ${ }^{1 *}$, \\ Huawei $\mathrm{Ma}^{2 *}$ and Dayan $\mathrm{Zhou}^{1}$ \\ ${ }^{1}$ Breeding Laboratory, Guangxi Aquatic Products Introduction and Breeding Center, 30 \\ Baisha Avenue, Jiangnan District, Nanning 530031, China. \\ ${ }^{2}$ Biotechnology Laboratory, Guangxi Academy of Fishery Sciences, No. 8 Qingshan \\ Road, Qingxiu District, Nanning 530021, China.
}

Jinzhao He, Pengfei Feng and Junqi Qin have contributed equally as first co-authors.

Supplementary Table I. KEGG pathway classification of contigs.

\begin{tabular}{|c|c|c|c|}
\hline Pathway (Up_regulated) & $\begin{array}{l}\text { Number of } \\
\text { contigs (n) }\end{array}$ & Pathway (Down_regulated) & $\begin{array}{l}\text { Number of } \\
\text { contigs (n) }\end{array}$ \\
\hline Carbon metabolism & 10 & Carbon metabolism & 1 \\
\hline 2 Oxocarboxylic acid metabolism & 1 & 2-Oxocarboxylic acid metabolism & 1 \\
\hline Fatty acid metabolism & 3 & Fatty acid metabolism & 2 \\
\hline Biosynthesis of amino acids & 9 & Biosynthesis of amino acids & 1 \\
\hline $\begin{array}{l}\text { Degradation of aromatic compounds; Glycolysis } \\
\text { Gluconeogenesis }\end{array}$ & 9 & $\begin{array}{l}\text { Degradation of aromatic compounds; Glycolysis } \\
\text { Gluconeogenesis; Citrate cycle TCA cycle; Pentose } \\
\text { phosphate pathway }\end{array}$ & 1 \\
\hline Citrate cycle TCA cycle & 2 & Pentose and glucuronate interconversions & 2 \\
\hline Pentose phosphate pathway & 5 & Fructose and mannose metabolism & 3 \\
\hline $\begin{array}{l}\text { Pentose and glucuronate interconversions; Fruc- } \\
\text { tose and mannose metabolism }\end{array}$ & 4 & $\begin{array}{l}\text { Galactose metabolism; Ascorbate and aldarate } \\
\text { metabolism; Starch and sucrose metabolism }\end{array}$ & 1 \\
\hline Galactose metabolism & 1 & Amino sugar and nucleotide sugar metabolism & 1 \\
\hline $\begin{array}{l}\text { Ascorbate and aldarate metabolism; Starch and } \\
\text { sucrose metabolism }\end{array}$ & 2 & $\begin{array}{l}\text { Pyruvate metabolism; Glyoxylate and dicarboxylate } \\
\text { metabolism; Propanoate metabolism; Butanoate } \\
\text { metabolism; C5 Branched dibasic acid metabolism; } \\
\text { Inositol phosphate metabolism }\end{array}$ & 1 \\
\hline Amino sugar and nucleotide sugar metabolism & 3 & Oxidative phosphorylation & 1 \\
\hline Pyruvate metabolism & 4 & $\begin{array}{l}\text { Photosynthesis; Photosynthesis antenna proteins; } \\
\text { Photosynthesis proteins; Carbon fixation in photo- } \\
\text { synthetic organisms; Carbon fixation pathways in } \\
\text { prokaryotes; Methane metabolism; Nitrogen metab- } \\
\text { olism; Sulfur metabolism; Fatty acid biosynthesis }\end{array}$ & 1 \\
\hline Glyoxylate and dicarboxylate metabolism & 3 & Fatty acid elongation & 2 \\
\hline
\end{tabular}

Table continue on next page

* Corresponding author: 260229251@qq.com; 0czg@163.

com; ma463543285@126.com

0030-9923/2022/0001-0001\$ 9.00/0

Copyright 2022 Zoological Society of Pakistan 


\begin{tabular}{|c|c|}
\hline Pathway (Up_regulated) & \\
\hline Propanoate metabolism & 1 \\
\hline $\begin{array}{l}\text { Butanoate metabolism; C5 Branched dibasic acid } \\
\text { metabolism; Inositol phosphate metabolism }\end{array}$ & 1 \\
\hline Oxidative phosphorylation & 7 \\
\hline $\begin{array}{l}\text { Photosynthesis; Photosynthesis antenna proteins; } 5 \\
\text { Photosynthesis proteins; Carbon fixation in photo- } \\
\text { synthetic organisms }\end{array}$ & 5 \\
\hline Carbon fixation pathways in prokaryotes & 1 \\
\hline Methane metabolism & 5 \\
\hline Nitrogen metabolism & 3 \\
\hline Sulfur metabolism & 1 \\
\hline Fatty acid biosynthesis;Fatty acid elongation & 8 \\
\hline Fatty acid degradation & 1 \\
\hline $\begin{array}{l}\text { Synthesis and degradation of ketone bodies; Cutin } 1 \\
\text { suberine and wax biosynthesis; Steroid biosynthe- } \\
\text { sis; Primary bile acid biosynthesis }\end{array}$ & \\
\hline
\end{tabular}

Secondary bile acid biosynthesis; Steroid hormone 9 biosynthesis

Glycerolipid metabolism

Glycerophospholipid metabolism

Ether lipid metabolism

Sphingolipid metabolism

Arachidonic acid metabolism

4

Linoleic acid metabolism; alpha Linolenic acid metabolism; Biosynthesis of unsaturated fatty acids

Lipid biosynthesis proteins; Purine metabolism

Pyrimidine metabolism

Alanine aspartate and glutamate metabolism

Glycine serine and threonine metabolism

Cysteine and methionine metabolism

Valine leucine and isoleucine degradation; Valine 2 leucine and isoleucine biosynthesis; Lysine biosynthesis; Lysine degradation

Arginine biosynthesis

Arginine and proline metabolism

Number of
contigs (n)

(n)

.

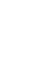

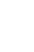




\begin{tabular}{llll}
\hline Pathway (Up_regulated) & $\begin{array}{l}\text { Number of } \\
\text { contigs (n) }\end{array}$ & Pathway (Down_regulated) & $\begin{array}{l}\text { Number of } \\
\text { contigs (n) }\end{array}$ \\
\hline $\begin{array}{l}\text { Histidine metabolism; Tyrosine metabolism; Phe- } \\
\text { nylalanine metabolism; Tryptophan metabolism }\end{array}$ & 3 & Mucin type O glycan biosynthesis & 1 \\
$\begin{array}{l}\text { Phenylalanine tyrosine and tryptophan biosyn- } \\
\text { (n) }\end{array}$ & Other types of O glycan biosynthesis & 2
\end{tabular}
thesis; Amino acid related enzymes; beta Alanine metabolism

Taurine and hypotaurine metabolism

Glycosaminoglycan biosynthesis chondroitin sul- 1 fate dermatan sulfate; Glycosaminoglycan biosynthesis heparan sulfate heparin; Glycosaminoglycan biosynthesis keratan sulfate; Proteoglycans; Glycosaminoglycan binding proteins; Glycosylphosphatidylinositol GPI anchored proteins; Glycosaminoglycan degradation

Phosphonate and phosphinate metabolism; Selen- 4 Glycosylphosphatidylinositol GPI anchor biosyn- 1 ocompound metabolism; Cyanoamino acid metabolism; D Glutamine and D glutamate metabolism; $\mathrm{D}$ Arginine and $\mathrm{D}$ ornithine metabolism; D Alanine metabolism; Glutathione metabolism

Glycosyltransferases; N Glycan biosynthesis; Var- 2 ious types of $\mathrm{N}$ glycan biosynthesis; Mucin type $\mathrm{O}$ glycan biosynthesis

Other types of O glycan biosynthesis; Glycosaminoglycan biosynthesis chondroitin sulfate dermatan sulfate; Glycosaminoglycan biosynthesis heparan sulfate heparin

Glycosaminoglycan biosynthesis keratan sulfate thesis; Glycosphingolipid biosynthesis lacto and neolacto series

Glycosphingolipid biosynthesis globo series;

Glycosphingolipid biosynthesis ganglio series; Lipopolysaccharide biosynthesis; Lipopolysaccharide biosynthesis proteins; Peptidoglycan biosynthesis; Other glycan degradation; Thiamine metabolism; Riboflavin metabolism; Vitamin B6 metabolism; Nicotinate and nicotinamide metabolism

Pantothenate and CoA biosynthesis; Biotin metab- 1 olism

Lipoic acid metabolism; Folate biosynthesis; One 3 carbon pool by folate; Retinol metabolism

Proteoglycans; Glycosaminoglycan binding pro- 1 teins; Glycosylphosphatidylinositol GPI anchored proteins; Glycosaminoglycan degradation; Glycosylphosphatidylinositol GPI anchor biosynthesis; Porphyrin and chlorophyll metabolism; Ubiquinone 1 and other terpenoid quinone biosynthesis; Prenyltransferases; Terpenoid backbone biosynthesis Glycosphingolipid biosynthesis lacto and neolacto series

Monoterpenoid biosynthesis; Sesquiterpenoid and $\quad 1$ triterpenoid biosynthesis 
Glycosphingolipid biosynthesis ganglio series 1

Lipopolysaccharide biosynthesis; Lipopolysaccharide biosynthesis proteins; Peptidoglycan biosynthesis; Other glycan degradation; Thiamine metabolism; Riboflavin metabolism

Vitamin B6 metabolism; Nicotinate and nicotinamide metabolism

Pantothenate and CoA biosynthesis; Biotin metab- 1 olism; Lipoic acid metabolism; Folate biosynthesis; One carbon pool by folate

Retinol metabolism

Porphyrin and chlorophyll metabolism; Ubiquinone and other terpenoid quinone biosynthesis; Prenyltransferases; Terpenoid backbone biosynthesis
Diterpenoid biosynthesis; Carotenoid biosynthe3 sis; Brassinosteroid biosynthesis; Insect hormone biosynthesis; Zeatin biosynthesis; Limonene and pinene degradation; Geraniol degradation; Polyketide biosynthesis proteins; Type I polyketide structures; Biosynthesis of 1214 and 16 membered macrolides; Biosynthesis of ansamycins; Biosynthesis of type II polyketide backbone; Biosynthesis of type II polyketide products; Tetracycline biosynthesis; Polyketide sugar unit biosynthesis; Nonribosomal peptide structures; Biosynthesis of siderophore group nonribosomal peptides; Biosynthesis of vancomycin group antibiotics; Phenylpropanoid biosynthesis; Stilbenoid diarylheptanoid and gingerol biosynthesis; Flavonoid biosynthesis; Flavone and flavonol biosynthesis; Anthocyanin biosynthesis; Isoflavonoid biosynthesis; Indole alkaloid biosynthesis; Indole diterpene alkaloid biosynthesis; Isoquinoline alkaloid biosynthesis Tropane piperidine and pyridine alkaloid biosynthesis; Acridone alkaloid biosynthesis; Caffeine metabolism; Betalain biosynthesis; Glucosinolate biosynthesis; Benzoxazinoid biosynthesis; Penicillin and cephalosporin biosynthesis; Carbapenem biosynthesis; Monobactam biosynthesis; Clavulanic acid biosynthesis; Streptomycin biosynthesis; Butirosin and neomycin biosynthesis; Puromycin biosynthesis; Novobiocin biosynthesis; Aflatoxin biosynthesis; Benzoate degradation; Aminobenzoate degradation; Fluorobenzoate degradation; Chloroalkane and chloroalkene degradation; Chlorocyclohexane and chlorobenzene degradation; Toluene degradation; Xylene degradation; Nitrotoluene degradation; Ethylbenzene degradation; Styrene degradation; Atrazine degradation; Caprolactam degradation; 111 Trichloro 22 bis 4 chlorophenyl ethane DDT degradation; Bisphenol degradation; Dioxin degradation; Naphthalene degradation; Polycyclic aromatic hydrocarbon degradation; Furfural degradation; Steroid degradation; Metabolism of xenobiotics by cytochrome P450; Drug metabolism cytochrome $\mathrm{P} 450$

Drug metabolism other enzymes; Enzymes; Protein 1 kinases; Protein phosphatase and associated proteins; Peptidases; Cytochrome P450; KEGG modules in global; RNA polymerase; Basal transcription factors

Transcription factors; Transcription machinery; Spliceosome

\section{Spliceosome; Ribosome}

1

Ribosome; Transfer RNA biogenesis; Aminoacyl tRNA biosynthesis; RNA transport; mRNA surveillance pathway 
Monoterpenoid biosynthesis; Sesquiterpenoid and 1 triterpenoid biosynthesis; Diterpenoid biosynthesis; Carotenoid biosynthesis; Brassinosteroid biosynthesis; Insect hormone biosynthesis; Zeatin biosynthesis; Limonene and pinene degradation; Geraniol degradation; Polyketide biosynthesis proteins; Type I polyketide structures; Biosynthesis of 1214 and 16 membered macrolides; Biosynthesis of ansamycins; Biosynthesis of type II polyketide backbone; Biosynthesis of type II polyketide products; Tetracycline biosynthesis; Polyketide sugar unit biosynthesis; Nonribosomal peptide structures; Biosynthesis of siderophore group nonribosomal peptides; Biosynthesis of vancomycin group antibiotics; Phenylpropanoid biosynthesis; Stilbenoid diarylheptanoid and gingerol biosynthesis; Flavonoid biosynthesis; Flavone and flavonol biosynthesis; Anthocyanin biosynthesis; Isoflavonoid biosynthesis; Indole alkaloid biosynthesis; Indole diterpene alkaloid biosynthesis; Isoquinoline alkaloid biosynthesis; Tropane piperidine and pyridine alkaloid biosynthesis; Acridone alkaloid biosynthesis; Caffeine metabolism; Betalain biosynthesis; Glucosinolate biosynthesis; Benzoxazinoid biosynthesis; Penicillin and cephalosporin biosynthesis; Carbapenem biosynthesis; Monobactam biosynthesis; Clavulanic acid biosynthesis; Streptomycin biosynthesis

Butirosin and neomycin biosynthesis; Puromycin biosynthesis; Novobiocin biosynthesis; Aflatoxin biosynthesis; Benzoate degradation

Aminobenzoate degradation; Fluorobenzoate degradation; Chloroalkane and chloroalkene degradation; Chlorocyclohexane and chlorobenzene degradation; Toluene degradation; Xylene degradation; Nitrotoluene degradation; Ethylbenzene degradation; Styrene degradation; Atrazine degradation; Caprolactam degradation; 111 Trichloro 22 bis 4 chlorophenyl ethane DDT degradation; Bisphenol degradation; Dioxin degradation; Naphthalene degradation; Polycyclic aromatic hydrocarbon degradation; Furfural degradation; Steroid degradation; Metabolism of xenobiotics by cytochrome P450

Drug metabolism cytochrome P450

Drug metabolism other enzymes;Enzymes; Protein kinases;Protein phosphatase and associated proteins; Peptidases; Cytochrome P450; KEGG modules in global; RNA polymerase; Basal transcription factors

Transcription factors; Transcription machinery; Spliceosome

Spliceosome; Ribosome
Messenger RNA Biogenesis; Ribosome biogenesis 1 in eukaryotes; Ribosome biogenesis; Mitochondrial biogenesis; Translation factors; Chaperones and folding catalysts; Protein export
Protein processing in endoplasmic reticulum

SNARE interactions in vesicular transport; SNAREs; Ubiquitin mediated proteolysis
Ubiquitin system; Sulfur relay system; Proteasome 3

Proteasome; RNA degradation; DNA replication 6

DNA replication proteins; Chromosome and associ- 2 ated proteins; Base excision repair

Nucleotide excision repair 
Ribosome; Transfer RNA biogenesis; Aminoacyl 2 tRNA biosynthesis; RNA transport

mRNA surveillance pathway

Messenger RNA Biogenesis; Ribosome biogenesis 8 in eukaryotes; Ribosome biogenesis; Mitochondrial biogenesis; Translation factors; Chaperones and folding catalysts; Protein export; Protein processing in endoplasmic reticulum

SNARE interactions in vesicular transport;

SNAREs; Ubiquitin mediated proteolysis

Ubiquitin system; Sulfur relay system; Proteasome 1

Proteasome; RNA degradation

DNA replication; DNA replication proteins; Chro- 3 mosome and associated proteins; Base excision repair; Nucleotide excision repair; Mismatch repair; Homologous recombination; Non homologous end joining; Fanconi anemia pathway; DNA repair and recombination proteins; Non coding RNAs; Transporters; $\mathrm{ABC}$ transporters; Phosphotransferase system PTS; Bacterial secretion system; Secretion system; Two component system

Two component system; Ras signaling pathway 4

Rap1 signaling pathway

MAPK signaling pathway

ErbB signaling pathway

Wnt signaling pathway

Notch signaling pathway; Hedgehog signaling

pathway; TGF beta signaling pathway

Hippo signaling pathway

VEGF signaling pathway

Jak STAT signaling pathway

NF kappa B signaling pathway

TNF signaling pathway

HIF 1 signaling pathway

FoxO signaling pathway

Calcium signaling pathway

Phosphatidylinositol signaling system

Phospholipase D signaling pathway

Sphingolipid signaling pathway

cAMP signaling pathway

cGMP PKG signaling pathway

PI3K Akt signaling pathway

AMPK signaling pathway

mTOR signaling pathway

西

2

3

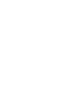


Plant hormone signal transduction; G protein cou- 2 pled receptors; Enzyme linked receptors; Cytokine receptors; Nuclear receptors; Ion channels; GTP binding proteins; Neuroactive ligand receptor interaction

Cytokine cytokine receptor interaction 7

Cytokines; ECM receptor interaction

Cell adhesion molecules CAMs

Cell adhesion molecules and their ligands; CD

Molecules; Lectins; Bacterial toxins; Endocytosis

Exosome; Phagosome

Lysosome

1

Peroxisome

2

Regulation of autophagy; Regulation of mitophagy 6 yeast; Prokaryotic Defense System; Bacterial chemotaxis; Bacterial motility proteins; Flagellar assembly; Regulation of actin cytoskeleton

Cytoskeleton proteins; Cell cycle

Cell cycle yeast; Cell cycle Caulobacter; Meiosis 8 yeast; Oocyte meiosis; Apoptosis

p53 signaling pathway

Focal adhesion

Adherens junction

Tight junction

Gap junction

Signaling pathways regulating pluripotency of stem cells; Hematopoietic cell lineage

Complement and coagulation cascades $\quad 4$

Platelet activation 2

Toll like receptor signaling pathway 3

NOD like receptor signaling pathway $\quad 7$

RIG I like receptor signaling pathway 3

Cytosolic DNA sensing pathway 1

Natural killer cell mediated cytotoxicity 4

Antigen processing and presentation
Cell adhesion molecules and their ligands; CD Mol- 14 ecules; Lectins; Bacterial toxins; Endocytosis

Exosome; Phagosome 12

Lysosome 2

Peroxisome 2

Regulation of autophagy; Regulation of mitophagy 6 yeast; Prokaryotic Defense System; Bacterial chemotaxis; Bacterial motility proteins; Flagellar assembly; Regulation of actin cytoskeleton

Cytoskeleton proteins; Cell cycle 9

Cell cycle yeast 4

Cell cycle Caulobacter; Meiosis yeast 4

Oocyte meiosis 3

Apoptosis 12

p53 signaling pathway 2 


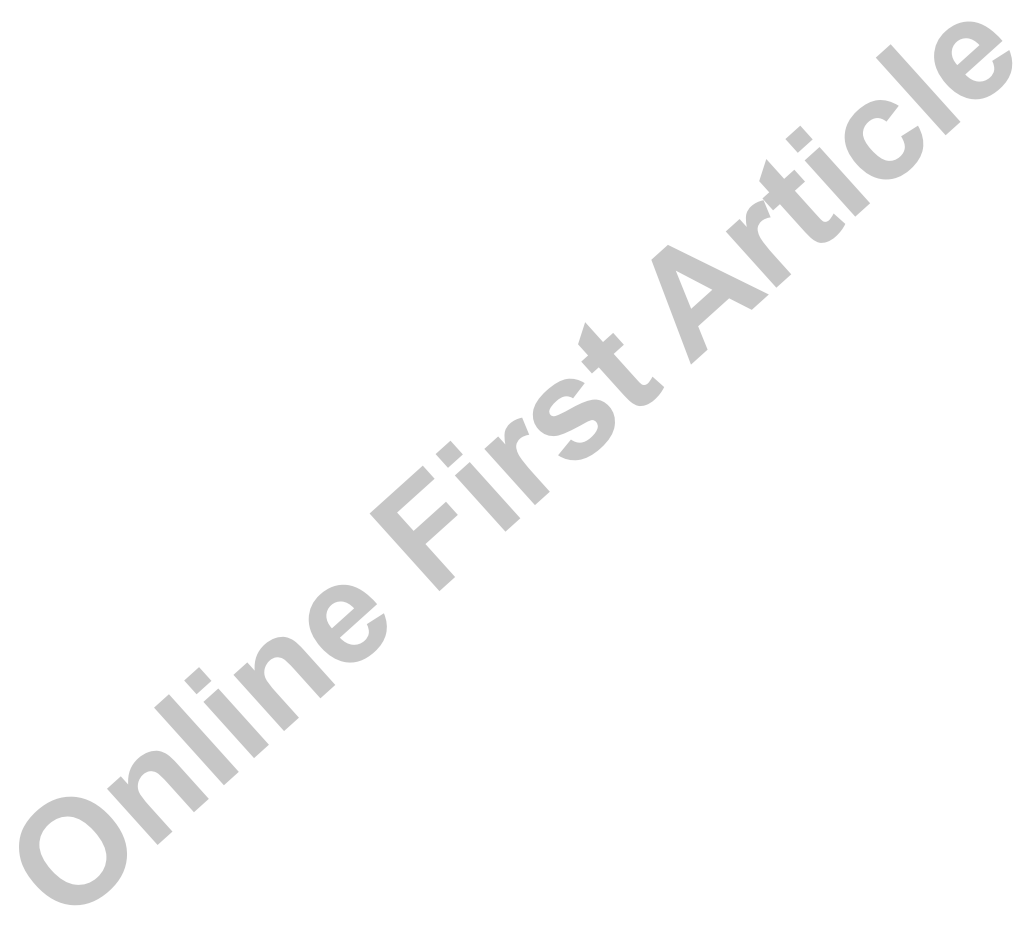


